ISBN 978-93-84422-76-9

6th International Conference on Developments in Engineering and Technology

(ICDET-2017)

Bangkok (Thailand) Feb.6-7, 2017

\title{
Visible Light Photocatalytic Degradation of Methylene Blue Using $\mathrm{V}_{2} \mathrm{O}_{5}$ Nanoparticles
}

\author{
Viruntachar Kruefu ${ }^{1}$, Hathaithip Sintuya ${ }^{2}$, Pusit Pookmanee ${ }^{3}$ and Sukon Phanichphant ${ }^{4}$ \\ ${ }^{1}$ Program in Nanoscience and Nanotechnology, Faculty of Science, Maejo University, \\ Chiang Mai, 50290 Thailand \\ ${ }^{2}$ Asian Development Colleges for Community Economy and Technology, Chiang Mai Rajabhat University, \\ Chiang Mai, 50300 Thailand \\ ${ }^{3}$ Program in Chemistry, Faculty of Science, Maejo University, Chiang Mai, 50290, Thailand \\ ${ }^{4}$ Materials Science Research Center, Faculty of Science, Chiang Mai University, 50200 Thailand
}

\begin{abstract}
Flame spray pyrolysis (FSP) was employed to synthesize vanadium pentoxide $\left(\mathrm{V}_{2} \mathrm{O}_{5}\right)$. The crystalline phase, morphology and size of the nanoparticles were characterized by XRD, FE-SEM, HR-TEM and EDXS. The specific surface area of the nanoparticles was measured by nitrogen adsorption (BET analysis). The XRD patterns showed that the nanoparticles had the orthorhombic phase of $\mathrm{V}_{2} \mathrm{O}_{5}$ with the JCPDS file No. 41-1426. The SEM image showed nanoparticles have clear spherical morphologies. The crystallite size of $\mathrm{V}_{2} \mathrm{O}_{5}$ spherical particles was in the range of 20-30 $\mathrm{nm}$. The photocatalytic activities of $\mathrm{V}_{2} \mathrm{O}_{5} \mathrm{NPs}$ were determined by studying the degradation of methylene blue under visible light irradiation.
\end{abstract}

Keywords: $\mathrm{V}_{2} \mathrm{O}_{5}$, nanoparticles, flame spray pyrolysis, methylene blue, visible light

\section{Introduction}

Vanadium pentoxide $\left(\mathrm{V}_{2} \mathrm{O}_{5}\right)$ nanoparticles (NPs) is an one kind of the important photocatalysts material because of its unique advantages, such as its low price, easy synthesis, availability, good optical and electrical properties. $\mathrm{V}_{2} \mathrm{O}_{5}$ NPs can be synthesized by different methods including sol-gel [1-2], electrospinning [3], hydrothermal [4], chemical vapour deposition [5] flame spray pyrolysis [6] and precipitation [7]. Flame spray pyrolysis (FSP) is a versatile process for synthesis of nanoparticles materials with high surface area [8].

Methylene blue (MB) is a commonly dye that finds enormous applications in dyeing industry. In order to treat the dye effluents photocatalysis is one of the feasible methodologies that can be effectively exploited for the complete degradation of various dye pollutants [9-11].

In this research, $\mathrm{V}_{2} \mathrm{O}_{5}$ NPs were synthesized by a one-step of flame spray pyrolysis (FSP) process. The crystalline phase, morphologies and particles size of the NPs were characterized by XRD, FE-SEM, HR-TEM and EDXS. The specific surface area of the nanoparticles $\left(S S A_{\mathrm{BET}}\right)$ was measured by nitrogen adsorption method and the effects of $\mathrm{V}_{2} \mathrm{O}_{5}$ catalyst on the photocatalytic decomposition behavior of methylene blue were investigated.

\section{Experimental}

\subsection{Preparation and characterization of the catalyst}

The $\mathrm{V}_{2} \mathrm{O}_{5}$ NPs were synthesized by FSP technique [7] by using vanadium (V) oxytripropoxide (Aldrich, $98 \%)$ diluted in diethylene glycol/toluene/tetrahydrofuran $(30 / 30 / 20 \mathrm{vol} \%)$ as the precursor solution. The solutions were fed into a FSP reactor by a syringe pump with a constant feed rate of $5 \mathrm{~mL} / \mathrm{min}$ while $5 \mathrm{l} / \mathrm{min}_{2}$ 
is being dispersed (5/5 flame). After evaporation and combustion of precursor solution, particles are formed by nucleation, condensation, coagulation and coalescence. Finally, the nanopowders were collected on glass microfiber filters with the aid of a vacuum pump. The crystalline phase, morphologies and particles size of flame-made NPs were analyzed by X-ray diffraction spectroscopy (XRD) using $\mathrm{CuK}_{\alpha}$ radiation at $2 \theta=20-80^{\circ}$ with a step size of $0.06^{\circ}$ and a scanning speed of $0.72^{\circ} / \mathrm{min}$, field emission-scanning electron microscopy (FESEM) and high resolution- transmission electron microscopy (HR-TEM) and and energy dispersive X-ray spectrometry (EDXS). The specific surface area of $\mathrm{V}_{2} \mathrm{O}_{5}$ NPs was measured by nitrogen adsorption (BET) analysis. The optical properties of $\mathrm{V}_{2} \mathrm{O}_{5}$ NPs were evaluated in term of UV-Vis absorption spectra at room temperature.

\subsection{Photocatalytic measurement}

$0.05 \mathrm{~g} \mathrm{~V}_{2} \mathrm{O}_{5} \mathrm{NPs}$ were suspended into a $500 \mathrm{~mL}$ beaker containing $250 \mathrm{ml}$ of $10 \mathrm{~g} / \mathrm{L}$ methylene blue (MB, $\mathrm{C}_{16} \mathrm{H}_{18} \mathrm{ClN}_{3} \mathrm{~S}$, Merck, Germany). After $\mathrm{V}_{2} \mathrm{O}_{5}$ NPs suspension was continuously stirred for 30 min under dark condition, $5 \mathrm{~mL}$ of sample was withdrawn from suspension. Then it was centrifuged in order to separate $\mathrm{V}_{2} \mathrm{O}_{5}$ NPs from MB solution. The initial concentration $\left(C_{0}\right)$ of MB in the solution was measured with an UV-VIS Spectrophotometer (U-2900, Hitachi, Japan). Subsequently, the remain suspension was illuminated with visible light using halogen lamp (Philips 50W, 12V). The distance between suspension and halogen lamp was $15 \mathrm{~cm}$. The photodegradation was carried out under magnetic stirring for $2 \mathrm{~h}$. At each interval of $10 \mathrm{~min}, 5 \mathrm{~mL}$ of the irradiated suspension was withdrawn and centrifuged. The residual concentration of MB in the solution was determined with an UV-VIS Spectrophotometer (U-2900, Hitachi, Japan). The photodegradation experiment was also conducted only in the presence of visible light without $\mathrm{V}_{2} \mathrm{O}_{5}$ NPs for the comparison purpose.

\section{Results and discussion}

\subsection{Structure and morphology of $\mathrm{V}_{2} \mathrm{O}_{5} \mathrm{NPs}$}

The phase and crystallinity structures of $\mathrm{V}_{2} \mathrm{O}_{5}$ NPs were carried out by XRD analysis as shown in Fig. 1 . The typical XRD patterns of $\mathrm{V}_{2} \mathrm{O}_{5}$ NPs was highly crystalline, and all the diffraction peaks can be confirmed to be orthorhombic crystalline phase of vanadium pentoxide match well with the JCPDS file no. 41-1426 (Space group: Pmmn, $\mathrm{a}=11.51 \AA, \mathrm{b}=3.565 \AA, \mathrm{c}=4.372 \AA$ ). The crystallite size of $\mathrm{V}_{2} \mathrm{O}_{5}$ NPs was calculated using Sherrer's equation to be $\sim 22 \mathrm{~nm}$. The specific surface area $\left(S S A_{\mathrm{BET}}\right)$ of $\mathrm{V}_{2} \mathrm{O}_{5} \mathrm{NPs}$ was measured by nitrogen adsorption and the results clearly showed that $S S A_{\mathrm{BET}}$ of $\mathrm{V}_{2} \mathrm{O}_{5} \mathrm{NPs}$ was $\sim 56 \mathrm{~m}^{2} / \mathrm{g}$. Accurate particle size and morphology of $\mathrm{V}_{2} \mathrm{O}_{5}$ NPs were further confirmed by TEM images.

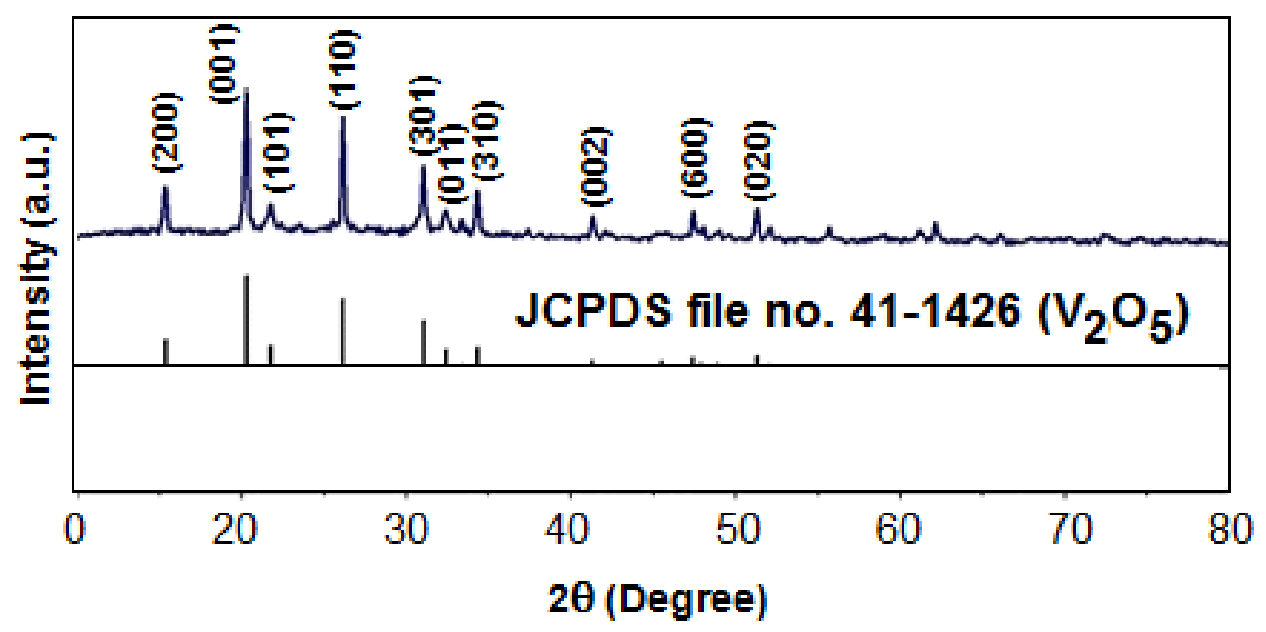

Fig. 1: XRD patterns of the flame-synthesis $\mathrm{V}_{2} \mathrm{O}_{5}$ NPs. 
Figure 2 (a) and (b) show the SEM micrographs and EDXS analysis of $\mathrm{V}_{2} \mathrm{O}_{5}$. The NPs had agglomerated nanosphere morphology with an average diameter of 20-40 nm. The chemical elements of $\mathrm{V}_{2} \mathrm{O}_{5}$ NPs were analyzed from EDXS spectra. The signal of EDXS spectra corresponded to V and O elements.
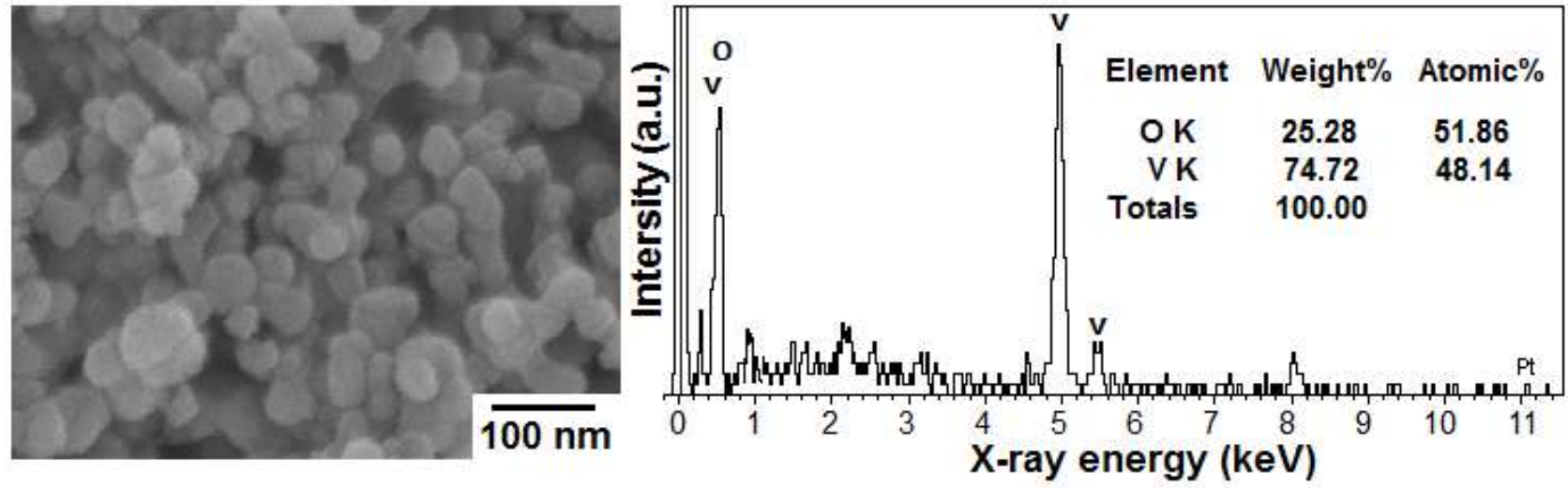

Fig. 2: (a) SEM image (b) EDXS spectra of $\mathrm{V}_{2} \mathrm{O}_{5}$ NPs

HR-TEM image of $\mathrm{V}_{2} \mathrm{O}_{5}$ is shown in Fig. 3a,b. Spherical $\mathrm{V}_{2} \mathrm{O}_{5}$ NPs (20-40nm) were found according to the TEM results. Figure $7 \mathrm{~b}$ indicates that the $d$-spacing was about $0.33 \mathrm{~nm}$ consistent with the (010) plane of the orthorhombic structure, indicating that these NPs grew along the [010] direction.

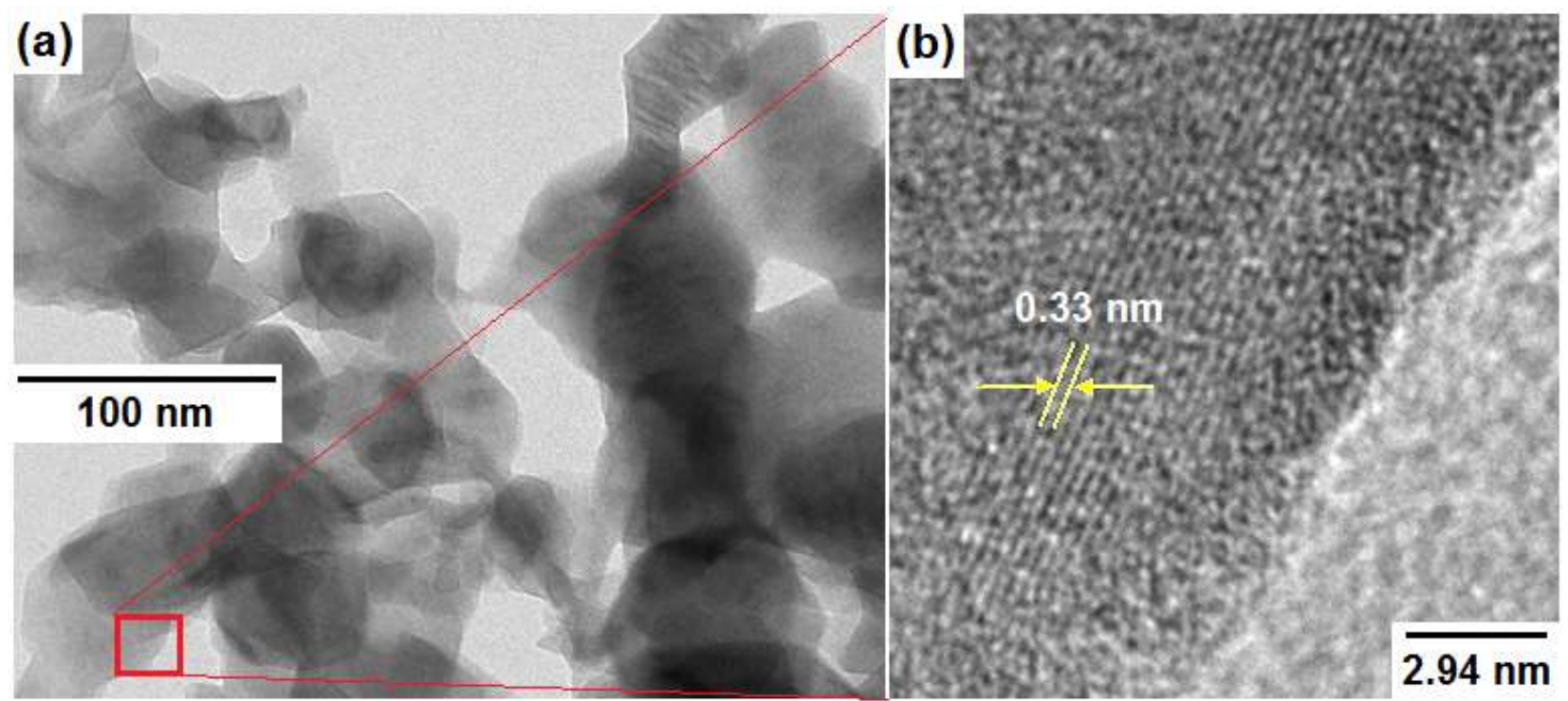

Fig. 3: (a) and (b) HR-TEM image of $\mathrm{V}_{2} \mathrm{O}_{5} \mathrm{NPs}$

Figure 4(a) shows UV-vis diffuse reflectance spectra analysis of $\mathrm{V}_{2} \mathrm{O}_{5}$ NPs. The direct band gap energy can be estimated from a plot of $(\alpha h v)^{1 / 2}$ versus photon energy $(h v)$ as shown in Fig. 4(b). The intercept of the tangent to the plot will give a good approximation of the band gap energy for the $\mathrm{V}_{2} \mathrm{O}_{5}$ [12]. The estimated band gap energies were $2.03 \mathrm{eV}$ for $\mathrm{V}_{2} \mathrm{O}_{5}$. 

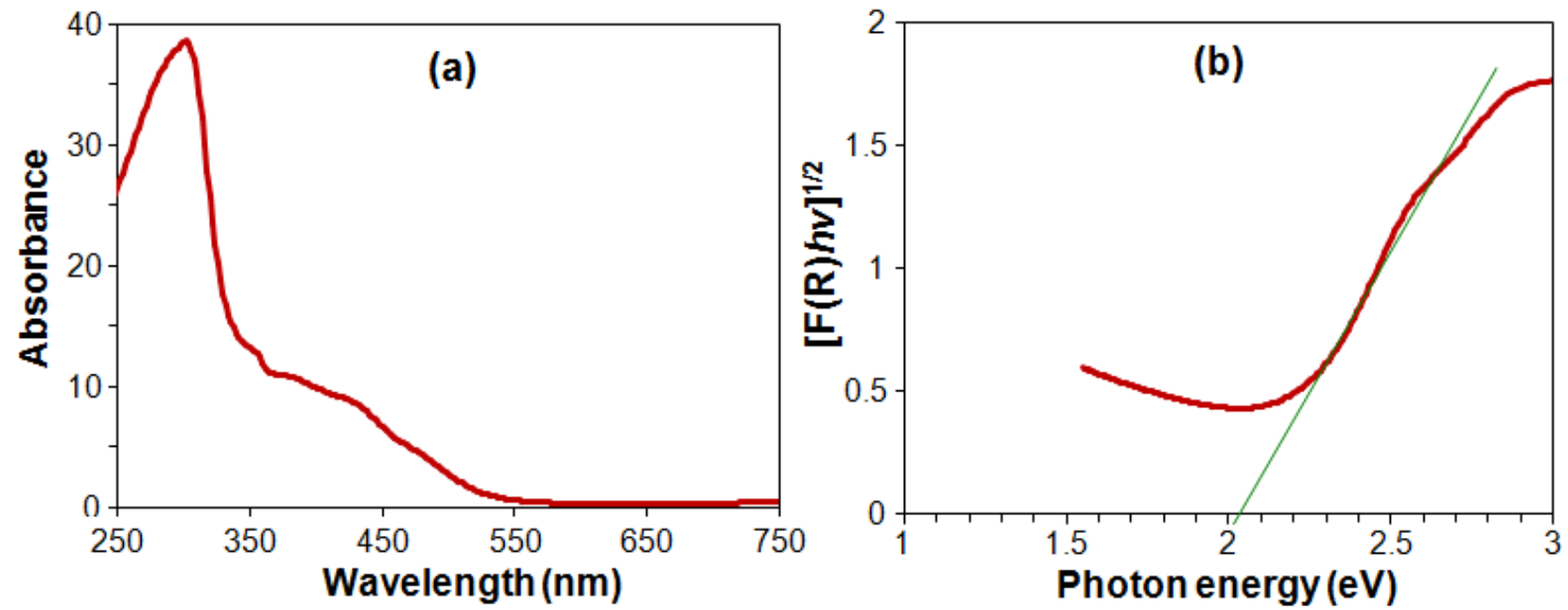

Fig. 4: (a) UV-vis diffuse reflectance spectra and (b) plots of $(\alpha h v)^{1 / 2}$ vs. photon energy $(h v)$ for $\mathrm{V}_{2} \mathrm{O}_{5}$ samples.

\subsection{Photocatalytic activity and mechanism}

Photodegradation of $\mathrm{MB}$ in the presence of $\mathrm{V}_{2} \mathrm{O}_{5}$ NPs synthesized via the flame spray pyrolysis method is shown in Figure 5(a). The results showed that ca. $81 \% \mathrm{MB}$ was degraded. For comparison purposes, the photodegradation experiment was also conducted only in the presence of visible light without $\mathrm{V}_{2} \mathrm{O}_{5}$ NPs. Without $\mathrm{V}_{2} \mathrm{O}_{5} \mathrm{NPs}$, only ca. $1 \% \mathrm{MB}$ was degraded. For the kinetic study, Figure 5(b) shows pseudo-first-order plot for MB degradation in the presence of $\mathrm{V}_{2} \mathrm{O}_{5}$ NPs synthesized via the flame spray pyrolysis method. The pseudo first-order model was explained by $\ln \left(C_{0} / C\right)=k t$ where $k$ is the apparent pseudo first-order rate constant $\left(\mathrm{min}^{-1}\right), C_{0}$ is the initial concentration of $\mathrm{MB}$, and $C$ is the concentration of $\mathrm{MB}$ at each interval of irradiation times $(t)$. It was found that a pseudo first-order rate constant $(k)$ for MB degradation in the presence of $\mathrm{V}_{2} \mathrm{O}_{5} \mathrm{NPs}$ was $0.0135 \mathrm{~min}^{-1}$.

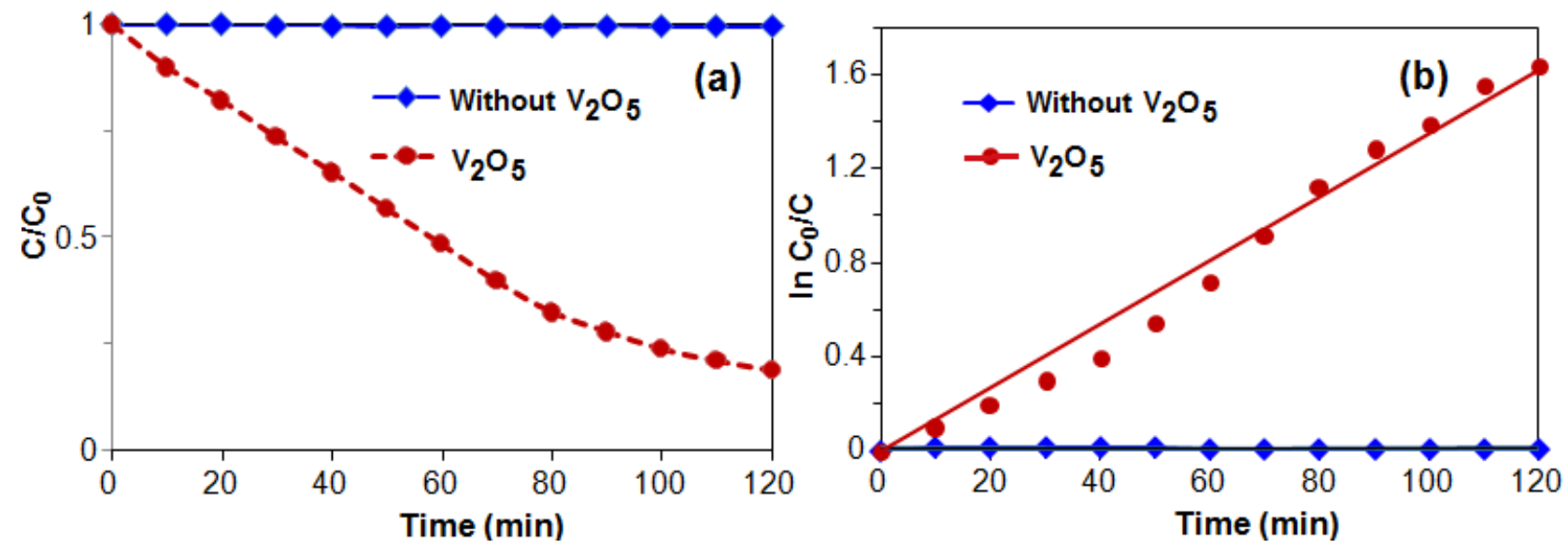

Fig. 5: (a) Photodegradation and (b) Pseudo-first-order plot for methylene blue degradation in the presence of $\mathrm{V}_{2} \mathrm{O}_{5} \mathrm{NPs}$ synthesized via the flame spray pyrolysis method.

\section{Conclusions}

In this research, $\mathrm{V}_{2} \mathrm{O}_{5}$ NPs were successfully synthesized by a one-step of flame spray pyrolysis (FSP) process. The XRD patterns showed that the nanoparticles had the orthorhombic phase of $\mathrm{V}_{2} \mathrm{O}_{5}$. The SEM image revealed nanoparticles have clear spherical morphologies. The crystallite size of $\mathrm{V}_{2} \mathrm{O}_{5}$ spherical particles was in the range of 20-30 nm. The $S S A_{\mathrm{BET}}$ of $\mathrm{V}_{2} \mathrm{O}_{5} \mathrm{NPs}$ was $\sim 56 \mathrm{~m}^{2} / \mathrm{g}$. The estimated band gap energies were $2.03 \mathrm{eV}$ for $\mathrm{V}_{2} \mathrm{O}_{5}$. Finally, the photocatalytic activities of $\mathrm{V}_{2} \mathrm{O}_{5} \mathrm{NPs}$ were determined by studying the degradation of MB under visible light irradiation. The results showed that ca. $81 \% \mathrm{MB}$ was degraded. $\mathrm{MB}$ degradation in the presence of $\mathrm{V}_{2} \mathrm{O}_{5}$ NPs was found to be pseudo-first-order reaction with the rate constant $(k)$ of $0.0135 \mathrm{~min}^{-1}$. 


\section{Acknowledgements}

The authors gratefully acknowledge the financial support from the National Research Council of Thailand (NRCT); Program in Nanoscience and Nanotechnology, Faculty of Science, Maejo University, Thailand; Program in Applied Physics, Faculty of Science, Maejo University, Thailand; Program in Chemistry, Faculty of Science, Maejo University, Thailand; Materials Science Research Center, Faculty of Science, Chiang Mai University, Thailand; Nanoscience and Nanotechnology Laboratory (NRL), Chiang Mai University, Thailand and Asian Development College for Community Economy and Technology, Chiang Mai Rajabhat University, Chiang Mai, 50300 Thailand.

\section{References}

[1] T. Puangpetch, S. Chavadej, and T. Sreethawong, "Mesoporous-assembled $\mathrm{V}_{2} \mathrm{O}_{5}$ nanosheet synthesized via a surfactant-modified sol-gel technique and its photocatalytic $\mathrm{H}_{2}$ production activity under visible light irradiation," Powder Technology, vol. 208, pp. 37-41, March 2011. https://doi.org/10.1016/j.powtec.2010.11.039

[2] V. Vivier, R. Baddour-Hadjean, J. P. Pereira-Ramos, and N. Baffier, "Barium vanadium pentoxide-based compounds prepared via a sol-gel process and their evaluation as lithium intercalation materials," J. Mater. Chem., Vol. 8, pp. 245-249, Jan 1998.

https://doi.org/10.1039/a707053e

[3] V. Modafferi, S. Trocino, A. Donato, G. Panzera, and G. Neri, "Electrospun $\mathrm{V}_{2} \mathrm{O}_{5}$ composite fibers:synthesis, characterization and ammonia sensing properties," Thin Solid Films, vol. 548, pp. 689-694, December 2013. https://doi.org/10.1016/j.tsf.2013.03.137

[4] J. Liu, X. Wang, Q. Peng, and Y. Li, "Vanadium pentoxide nanobelts; highly selective and stable ethanol sensor materials," Adv. Mater., Vol. 17, pp. 764-767, March 2005.

https://doi.org/10.1002/adma.200400993

[5] Y. Wang, Q. Su, C.H. Chen, M. L. Yu, G. J. Han, G. Q. Wang, K. Xin, W. Lan, and X. Q. Liu, "Low temperature growth of vanadium pentoxide nanomaterials by chemical vapour deposition using $\mathrm{VO}$ (acac)2 as precursor," J. Phys. D Appl. Phys., Vol. 43, pp. 185102, April 2010 https://doi.org/10.1088/0022-3727/43/18/185102

[6] S. H. Ng, T. J. Patey, R. Büchel, F. Krumeich, J. Z. Wang, H. K. Liu, S. E. Pratsinis, and P. Novák, "Flame spraypyrolyzed vanadium oxide nanoparticles for lithium battery cathodes," Phys. Chem. Chem. Phys., Vol. 11, pp. 37483755, March 2009. https://doi.org/10.1039/b821389p

[7] K. Shimizu, I. Chinzei, H. Nishiyama, S. Kakimoto, S. Sugaya, W. Matsutani, and A. Satsuma, "Doped-vanadium oxides as sensing materials for high temperature operative selective ammonia gas sensors," Sens. Actuators B Chemical, vol. 141, pp. 410-416, September 2009. https://doi.org/10.1016/j.snb.2009.06.048

[8] V. Kruefu, A. Wisitsoraat, and S. Phanichphant, "Enhanced ethanol selectivity of flame-spray-made Au/ZnO thick films," J. Nanosci. Nanotechnol., Vol. 14, pp. 7768-7773, October 2014. https://doi.org/10.1166/jnn.2014.9430

[9] X. Wan, M. Yuan, S.L. Tie, and S. Lan, "Effects of catalyst characters on the photocatalytic activity and process of $\mathrm{NiO}$ nanoparticles in the degradation of methylene blue," Appl. Surf. Sci., vol. 277, pp. 40-46, July 2013. https://doi.org/10.1016/j.apsusc.2013.03.126

[10] T. J. Whang, M. T. Hsieh, and H. H. Chen, "Visible-light photocatalytic degradation of methylene blue with laserinduced Ag/ZnO nanoparticles,” Appl. Surf. Sci. vol. 258, pp. 2796-2801, January 2012. https://doi.org/10.1016/j.apsusc.2011.10.134 
[11] R. Saravanan, H. Shankar, T. Prakash, V. Narayanan, and A. Stephen, "ZnO/CdO composite nanorods for photocatalytic degradation of methylene blue under visible light," Mater. Chem. Phys., Vol. 125, pp. 277-280, January $\underline{2011 .}$ https://doi.org/10.1016/j.matchemphys.2010.09.030

[12] W. A. Jr., C. Ribeiro, E. R. Leite, and V. R. Mastelaro, "Vanadium Pentoxide Nanostructures: An effective control of morphology and crystal structure in hydrothermal conditions," Crystal Growth \& Design, vol. 9, pp. 3626-3631, May $\underline{2009}$.

https://doi.org/10.1021/cg900373f 